\title{
The evaluation of the ideal athletic performance in terms of the absolute angles
}

\begin{abstract}
The scientific development that the sports events witness in the present time is strongly related to the technological development. Thus, the modern technical means such as developed devices and programs contributed in adopting scientific ways that lead to the finding of the most suitable, appropriate and economical kinesthetic paths for the athlete's body, using the best and modern ways of the kinesthetic analysis, which has a big role in diagnosing the mistakes and weakness and strength points of the athletes, for the fact that trainers base their work only on monitoring will not lead to the sufficient diagnosis of the mistakes, due to the speed of the movement during the performance, which leads also to the recognition of the effects of athletic training on the development of the physical abilities and applicable and mechanical indicators... etc. the aim of this research is to shed the light on the evaluation of the ideal athletic performance in terms of the absolute angles from a biomechanical perspective. Thus, through the recognition of the notion of the absolute angles and the transmission of the movement between them. Also knowing their relationship to the central and noncentral contraction of the muscle. Finally, we reached the following:

i. The fulfillment of the required torque is related to the principle of the central contraction of the muscle, which is related to the main duty of the movement.

ii. Whether the angle is relative or absolute, torque is the reason that the body or parts of body have (speed, acceleration and angular momentum)

The most important recommendation: it is obligatory not to name the angle by the name of the joint itself only, because the joint is the axis of the rotation that the body or part of the body rotate around it.
\end{abstract}

Keywords: athletic performance, absolute angles

\section{Introduction}

The development happening in the standard records and the high level of achievement that we see in the international championships is a result of the common scientific efforts of the experiences and the contributing sciences in the support of the athletic achievement. Here the importance of this research show up as an attempt to analyze the athletic performance and set the modern notions in the athletic training field, that aim at evaluating and defining the most important mistakes to subjectively correct the performance in terms of the absolute angles of body parts. Thus, they clarify the extent to which the athlete benefits from his abilities and understands the methods and ways of the performance in the practiced event, all that is to approach the ideal level and raise up to the best record (achievement).

In this scientific paper, we aim at explaining the following elements and clarifying their intertwining features and their effects and impact of one another:

1. The notion of the absolute angles.

2. The absolute angles and the transmission of the movement.

3. The absolute angles and the evaluation of the ideal performance.

4. The results.

\section{The notion of the absolute angles}

Many books and foreign and Arabic resources mentioned the notion absolute angles, all explained it their own point of view without addressing the extent to which it is used in the applicable field, so it is necessary to clarify the notion of this term so that researchers may use it correctly.

\author{
Volume 4 Issue I - 2019
}

\author{
Bouhadj Meziane, Baouche Khaled, Lebboukh \\ Toufik \\ Institute of Science and Techniques of Physical and Sports \\ Activities, University of Bouira,Algeria
}

Correspondence: Baouche Khaled, Institute of Science and Techniques of Physical and Sports Activities, University of Bouira,Algeria, Email khaled.baouchestaps@yahoo.com

Received: December 06, 2018 | Published: January 22, 2019
Dr. Sarih Abdul-Karim Al-Fadhli ${ }^{1}$ mentioned the identification of the term absolute angles that they are the vertical, horizontal and deep axes which are virtual lines that are vertical on the weight center of the body during the ordinary standing of the person. ${ }^{2}$ Those axes are also vertical on the different joints of the body during the movement of the related body parts, and we can attribute the movement of the linked parts with those joints to those axes. Which means that body parts rotate around those joints, which are considered as the rotation axes. When we say the knee joint, for example, we mean the rotation axes around which only the leg rotates. In addition, when we say the hip joint, we mean the rotation axis that the thigh rotates around it when the stem is fixed, and the rotation axis of the stem when the thigh is fixed, and so on for the rest of joints (rotation axes) that become the weight point center of the body or the center of weight of the other parts of the body rotate around these joints that are rotation axes.

1. Can we name the angle by the rotation axis, for example, we say the elbow angle, or knee angle or the hip angle?

2. Can we call the term "angular velocity" in the name of the joint, such as the angular velocity of the knee, elbow, or hip?

3. Can we measure the peripheral velocity for the end of the arm when it moves in the spiking skill for example indicating to the total arm length and its angular velocity knowing that there is changing in the arm length according to the performance steps?

4. What is the relationship of the movement of a body part around a certain joint with the central and non-central contraction?

5. Do the central and non-central contraction have a relationship with the functioning and ability of the working muscle? 


\section{All these questions need scientific answers.}

to answer this subject we should talk about the absolute angles:

scientists and researchers agreed on naming the angle that a part of body makes which relates to a common joint with another part, this angle is measured according to the vertical axes that meet in the same joint, on the condition that the other part that is related to the joint itself should be fixed in order that the first part moves by the absolute angle for the body part and the angle is name by that part (such as: thigh angle, stem angle, leg angle, ankle angle...) this naming means the absolute angle of that part that rotates around the near joint without the indication to the other part that is related to this joint. Often, the muscular functioning relates to these parts through those angles by the muscular torque that is resisting to the mass of that part that is related by the gravity or the weight added to it. Therefore, we can say that the training of the absolute torques of these parts which are considered also as main trainings for the development of the angular muscular strength that works on the rotation of these parts during the performance of the different skills( training the strength according to the movement of the body parts in the related skill), which means when those parts rotate around those joints with the fixing of the other part $^{3}$ Susan Hill ${ }^{4}$ added that the absolute angles are "the inclination or angular flexion of a part of the body according to the fixed indicator line, and they are important angles that indicate often to the deviation of the body parts themselves, so, when the stem is in the state of bending, the deviation angle of the stem affects directly on the amount of strength that should be generated through the muscle of the stem extension to support the stem in the required state. The bending angle of a part of body indicates to its total or absolute angle and it is measure according to the absolute or total vertical or horizontal indicator line, thus, the total absolute angles are measured by the same direction of the individual indicator whether vertically or horizontally" 4 Sarih abdel karim mentioned that the absolute angle is "the angle that a part of body crosses that relates to a joint shared with another part and this angle is measured according to the vertical axes on the same joint, and it is achieved through moving the joints that rotate around the first part with the condition that the other part that is linked with the same joint should be fixed in order that the first part moves, and it is an angular inclination or bending of a part of body according to the virtual vertical or horizontal coordination axes". ${ }^{2}$

Therefore, it should not name the angle by the name of the joint itself only, because the joint is the rotation axis that the body or body part rotate around it.

\section{The absolute angles and the transmission of the movement}

The muscular torque is calculated by (the muscle strength $\mathrm{x}$ the distance of its direction line from the joint) which is responsible for the movement of body part around the joint, that is, that torque deals with the part mass and length to achieve angular angle or angular acceleration according to the second law of Newton of the angular angles:

As: Strength $\mathrm{x}$ distance $\mathrm{x}$ pushing time $=$ final angular momentum - initial angular momentum

So: Momentous pushing torque $=$ the change in the angular momentum

Whether the angle is absolute or relative, the torque causes the body or part of body to have angular (speed, acceleration, or momentum) the symmetry of the torques values between the body parts means the symmetry of the transmission of the angular movement and the angular momentum between these parts. Therefore, the angular or linear momentum indicator and their transmission between the parts of the body by approximatively equal values can indicate to an ideal movement transmission between these parts and this indicator is considered as a quantitative measure for the kinesthetic transmission and not qualitative as the kinetic learning scientists say.

This principle leads us to the trainings of the absolute and relative strength according to those angles, thus, those trainings are trainings of absolute and relative torques, the absolute torques relate to the absolute angles and the relative torques relate to the relative angles.

The achievement of the required torque is related to the principle of the central contraction of the muscle that relates to main duty of the movement. Which means the body or body part should take the suitable position to prepare the muscle for the performance of the non-central contraction (negative) then the performance of a central contraction that is always positive, that what achieves the total torque of any group of torques. This requires the participation of all parts of the body that have the ability to participate with their extreme efforts. That is, the total result of the torques represents the sum of the maximum limits of the parts torques in order to achieve the aim of the skill. This means the participation of the biggest torque then the least one through the grading of the movement from the biggest mass to the least mass.

\section{The absolute angles and the evaluation of the ideal performance}

The values of the absolute angles of the body parts at the end of the execution of the main section may be an indicator for the ideal performance if they agree with the angle of the body starting, Figure 1 shows the ideal absolute angles for the starting of the body, and any odd value of these values in any part indicate a bad (weak) performance.

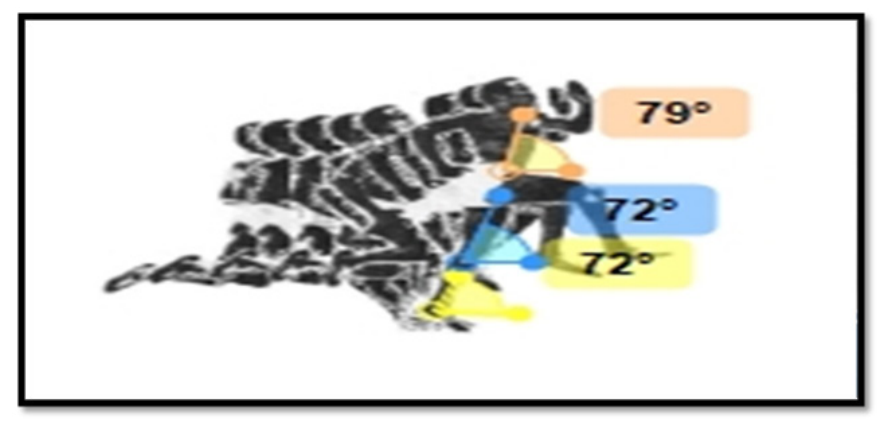

Figure I The perfection of the absolute angles of the functioning body parts in the final section of the movement indicates the perfection of the performance towards the movement.

\section{Results}

After looking at the previous elements, we deduced the following:

Achieving the required torque is related to the principle of the central contraction of the muscle that is related to the main duty of the movement.

Whether the angle is absolute or relative, the torque causes the body or body part to have (velocity, acceleration and angular momentum). 
Symmetry of the torques values between the body parts means the symmetry of the transmission of the angular velocity between these parts.

The body or part of body should take the suitable position to prepare for the non-central contraction (negative) then the performance of an always positive central contraction, and this what achieves the torque resulted for any group of the torques.

The absolute angles values for the body parts are an indicator for the ideal performance at the end of the execution the final section of the movement if they agreed with the angle of the body starting.

\section{The most important recommendation}

i. The angle should not be name by the name of the joint itself only, because the joint is the rotation axis that the body or body parts rotate around it.

ii. Trainers should be aware of the fact that the absolute torques relate to the absolute angles and the relative torques relate to the relative angles.

\section{Acknowledgments}

None.

\section{Conflicts of interest}

The authors declare no conflicts of interest.

\section{References}

1. Sarih Abdul Karim. Analysis of movement and practical application of axes, surfaces and angles and muscular work, the second lecture for doctoral students, Baghdad university college of physical education and sports sciences; 2016.

2. Sarih Abdul Karim. clarifying some of the mechanical concepts associated with sports science, a qualitative lecture presented to the first scientific conference - Faculty of education department of physical education and sports sciences - University of Kufa; 2016.

3. Louay Ghanem Al-Sumaida'i. Biomechanics and sports, Dar Al-Kuttab for Printing and Publishing, University of Mosul; 1987.

4. Susan Hill. Fundamentals of biomechanics: Translation, Hassan Hadi Zeidi. Baghdad, Book House and Documents; 2014. 